\title{
Distributed Extremum Seeking and Formation Control for Nonholonomic Mobile Network
}

\author{
Chaoyong Li, Zhihua Qu, and Mary Ann Weitnauer
}

\begin{abstract}
In this paper, an integrated control and optimization problem is studied in the context of formation and coverage of a cluster of nonholonomic mobile robots. In particular, each communication channel is modeled by its outage probability, and hence, connectivity is maintained if the outage probability is less than a certain threshold. The objective of the communication network is to not only maintain resilient communication quality but also extend the network coverage. An information theory based performance index is defined to quantify this control objective. Unlike most of the existing results, the proposed cooperative control design does not assume the knowledge of any gradient (of the performance index). Rather, a distributed extremum seeking algorithm is designed to optimize the connectivity and coverage of the mobile network. The proposed approach retains all the advantages of cooperative control, and it can not only perform extremum seeking individually, but also ensures a consensus of estimates between any pair of connected systems. Simulation results demonstrate effectiveness of the proposed methodology.
\end{abstract}

Chaoyong Li is with Intelligent Fusion Technology, Inc, Germantown, MD, 20876 USA. Chaoyong. 1 i@ucf . edu

Zhihua Qu is with Dept. Electrical Engineering and Computer Science, University of Central Florida, Orlando, FL, 32816, USA. Queucf.edu. His work is supported in part by US National Science Foundation under grants ECCS-1308928 and CCF-0956501, by US Department of Energy's award DE-EE0006340, by US Department of Transportation's award DTRT13-G-UTC51, by L-3 Communication's contract 11013I2034, and by Leidos' contract P010161530.

Mary Ann Weitnauer is with School of Electrical and Computer Engineering, Georgia Institute of Technology, Atlanta, Georgia 30332, USA. Mai@gatech. edu

A preliminary and abridged version of this paper [1] was presented at 50th IEEE Conference on Decision and Control, Orlando, FL, 2011 
integration leads to an inherent dilemma. Specifically, communication quality and network connectivity 2 favor agents/vehicles that are close and are moving closer, while network coverage demands them to 3 stay separated and even further apart. In other words, each agent needs to make its decision, preferably 4 distributively, to balance communication quality, connectivity, and network coverage such that overall 5 performance can be optimized.

In general, formation control can be categorized further into position based formation and distance based formation [3], the former is devoted to maintaining a specific relative geometric configuration [2][4], while 8 the later considers only the relative distance and bearing information [5] and is studied in the context of graph rigidity [6]. In this regard, coverage control [7] or flocking [8][9] can also be treated as special 10 cases of distance-based formation control, and the majority of existing results use either a potential field function or its variations to achieve a desired formation as well as to avoid collision [10]. However, singular configuration (i.e., local minimum) is inevitable in any application of these approaches [9], and common solutions to this problem employ some mild assumptions on either velocity [11] or explicit knowledge 14 of the leader [8]. In addition, formation control of nonholonomic systems has also received considerable 15 attentions. Specifically, I/O linearization for formation control of nonholonomic robots under a digraph 16 is introduced in [5], and a synchronization approach is proposed in [4] to study time varying formation 17 control of mobile robots. Other notable contributions include [12][13][14], where a hierarchical (three 18 layer) control conjecture is presented in [12] for coordination of mobile robots, and formation control and trajectory following of unicycles using saturated control scheme is studied in [13], while a distributed virtual structure approach is proposed in [14], all of which show global stability.

Mobile platforms are often equipped with wireless communication capabilities to provide and maintain connectivity of the communication network. To analyze this, modeling of communication channel quality is expected. For instance, metrics of communication quality such as SNR (i.e., signal-to-noise ratio) or Shannon capacity [15] are used for online measurements so that the current formation configuration (or relative distances) can be evaluated. As such, formation control can be accomplished by using communication quality as feedback instead of position information. Moreover, as shown in [16], the quality of a wireless communication link in a vehicular ad hoc network can be estimated by examining received data packets. In [17], motion control of networked robotic routers is investigated to maintain connectivity of a single user to a base station, which could be either stationary or adversarial. Recent advances on this topic include motion planning and gradient-based control of a robotic sensing network [18] to improve communication quality, optimization of SISO (i.e., single-input-single-output) communication chain under the assumption that gradient of SNR field is known [19], an online planing method is introduced in [20] to find a navigation path to meet network connectivity and bandwidth requirement, and an opportunistic 
communication strategy with energy constraint can be found in [21]. One closely related result can 2 be found in [1], where formation control of single-integrator system is investigated with the help of 3 classical extremum seeking scheme. However, there are the following key shortcomings in most of the 4 existing studies: absence of an analytical investigation of integrating communication and control issues in 5 nonholonomic mobile network, requirement of online extremum seeking algorithm with the knowledge 6 of gradients, and possible inconsistency of an extremum seeking scheme in multi-agent scenario.

In this paper, a distance based formation control scheme is introduced to separate each pair of connected vehicles with a specified/optimized distance, which represents the aforementioned tradeoff between communication quality and network coverage. The optimal distance can be estimated using a model-free and distributed extremum seeking scheme. Note that the classical extremum seeking scheme needs to be enhanced when applied to networked control systems, where estimates between any pair of connected systems are expected to be consistent. If consistency is not ensured, no stable formation can be achieved.

The contribution of this paper is twofold: (i) a new distance based formation strategy is proposed for nonholonomic robots that admits a communication performance metric; (ii) a new distributive extremum seeking scheme is designed to not only estimate the desired separation with acceptable accuracy but also ensure a consensus among estimates.

\section{Preliminaries}

\section{A. Graph theory}

In this paper, we consider an undirected graph $\mathscr{G}=(V, E)$, where $V=\{1,2, \ldots, n\}$ and $E$ denote 20 the sets of vertices/nodes and edges, respectively. Unless otherwise specified, vertex $j$ is said to be 21 adjacent/connected to vertex $i$ if $(j, i) \in E$, or equivalently $(i, j) \in E$. Analogously, neighborhood set $22 \mathscr{N}_{i} \subseteq V$ of vertex $i$ is $\{k \in V \mid(k, i) \in E\}$, the set of all vertices that are adjacent to vertex $i$. If $j \in \mathscr{N}_{i}$, 23 then $i \in \mathscr{N}_{j}$ holds as well.

Without loss of any generality, adjacency matrix $A(\mathscr{G})$ considered in this paper is weighted and 25 normalized as

$$
[A(\mathscr{G})]_{i k}= \begin{cases}a_{i k}>0 & \text { if }(k, i) \in E \\ 1-\sum_{k \neq i} a_{i k}>0 & \text { if } k=i \\ 0 & \text { otherwise }\end{cases}
$$

${ }_{26}$ That is, $A(\mathscr{G})$ is designed to be nonnegative, row-stochastic, and positive semi-definite. Furthermore, all its nonzero and hence positive weighting factors are both uniformly lower and upper bounded, i.e., $a_{i j} \in[\underline{a} 1]$, where $0<\underline{a}<1, \forall j \in \mathscr{N}_{i}$. Moreover, $\mathscr{G}$ is connected if its corresponding $A(\mathscr{G})$ is irreducible [2]. In 
1 addition, matrix $S(\mathscr{G})$ is defined as the connectivity matrix for graph $\mathscr{G}$, that is

$$
[S(\mathscr{G})]_{i j}=\left\{\begin{array}{cc}
1 & \text { if }(i, j) \in E \\
0 & \text { otherwise }
\end{array}\right.
$$

2 In order to accommodate the switching topologies, we define the time sequences $\left\{t_{k}: k \in \mathfrak{\aleph}\right\}$ for $\boldsymbol{\aleph}=$ $3\{0,1, \ldots, \infty\}$, and $\mathscr{G}$ is assumed to be time invariant during each of the intervals in the form of $\left[t_{k}, t_{k+1}\right)$, 4 and its corresponding adjacency matrix $\mathscr{A}$ is piecewise continuous as a result. That is, $\mathscr{G}\left(t_{k}\right)=\mathscr{G}\left(t_{k+1}^{-}\right)$. 5 Before proceeding further, the following assumption is stated regarding the connectivity and switching of 6 the underlying network.

$7 \quad$ Assumption 1: $\mathscr{G}$ is initially connected at time $t_{0}$ in the sense that there is an undirected link between 8 nodes $i$ and $j$ for all $i \neq j$ in $\mathscr{G}$.

\section{B. Outage probability of SISO communication}

In a mobile ad hoc network, communication quality of service (measured at the physical layer by 1 packet error rate, or outage rate between a transmitter and a receiver) depends on many unknown 2 parameters beyond their relative position such as multipath fading, shadowing, noise, and interfering. In 3 particular, the Shannon-Hartley law provides the relationship between distance and communication quality 14 when combined with the empirical radio propagation model [15]. In what follows, outage probability is introduced to quantitatively characterize the implications of distance $r_{i j}$ to data rate $\delta$, whose value is commonly used as the criterion to determine whether a viable communication channel is plausible between any given pair of mobile nodes. Specifically, for a SISO communication link [22],

$$
P\left[C_{S I S O}<\delta\right]=1-\exp \left(-\left(2^{\delta}-1\right) \frac{\sigma^{2}}{P_{0}}\left(\frac{r_{i j}}{r_{0}}\right)^{v}\right),
$$

where $C_{S I S O}$ is the Shannon capacity, $P_{0}$ is the transmitting power, $\sigma$ is the noise variance, $P_{0} / \sigma^{2}$ denotes SNR (i.e., signal-noise-ratio) at a reference distance, $v$ is the path loss exponent, $r_{0}$ is the reference distance from the transmitter to the receiver, and $r_{i j}$ is the effective distance.

The outage probability in (3) has the intuitive behavior that, as $r_{i j}$ grows, $P\left[C_{S I S O}<\delta\right] \rightarrow 1$ and, as $r_{i j} \rightarrow 0, P\left[C_{S I S O}<\delta\right] \rightarrow 0$, which is shown in figure 1 . In order to establish a viable communication, outage probability should always be less than certain threshold $\zeta$. That is,

$$
1-\exp \left(-\left(2^{\delta}-1\right) \frac{\sigma^{2}}{P_{0}}\left(\frac{r_{i j}}{r_{0}}\right)^{v}\right) \leq \frac{\zeta}{100} .
$$

24 Solving (4) for $r_{i j}$ yields the maximum distance between transmitter and receiver that will give the worst outage probability of $\zeta \%$, which is referred to as $r_{x}$. In other words, any communication beyond $r_{x}$ will be too corrupted to be considered effective, due to the signal drop rate and data outage. Taking figure 1 for instance, $r_{x}=68, r_{x}=105$, and $r_{x}=150$ correspond to outage probability being $9 \%$ (point $A$ ), $30 \%$ (point $B$ ), and $60 \%$ (point $C$ ), respectively. 


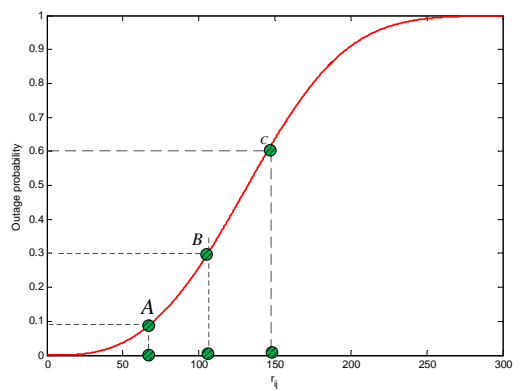

Fig. 1: Outage probability $P\left[C_{S I S O}<\delta\right]$ with $\delta=2, v=3, r_{0}=1, P_{0} / \sigma^{2}=10^{7}$

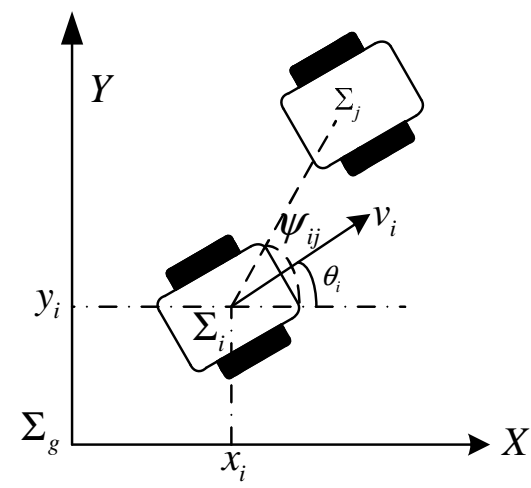

Fig. 2: Nonholonomic robots

\section{Problem Formulation}

2 Consider a group of $n$ nonholonomic mobile robots performing formation mission in a plane. For each 3 robot $i \in\{1,2, \ldots, n\}$, we denote $p_{i}=\left[x_{i} y_{i}\right]^{T}$ as its position in the $X-Y$ frame and $\theta_{i}$ as its current heading 4 angle with respect to the $x$-axis, as illustrated in figure 2. Thus, the kinematic equations of robot $i$ are:

$$
\left[\begin{array}{c}
\dot{x}_{i} \\
\dot{y}_{i} \\
\dot{\theta}_{i}
\end{array}\right]=\left[\begin{array}{c}
0 \\
0 \\
1
\end{array}\right] \omega_{i}+\left[\begin{array}{c}
\cos \theta_{i} \\
\sin \theta_{i} \\
0
\end{array}\right] v_{i},
$$

5 where $v_{i}$ and $\omega_{i}$ are the control inputs to be designed, detailed stability and control analysis of nonholo-

6 nomic systems can be found in [23].

7 In this paper, a distributed formation control scheme is proposed to drive all $n$ robots to a formation

8 in which the robots are separated by the optimized distance for both neighboring communication quality

9 and network coverage. In other words, distance based formation control is studied with consideration of

10 communication quality and network coverage. The technical problems to be investigated are summarized 11 as follows: 
Problem 1: (Distance based formation): Design a distributed control scheme such that, for any $j \neq i$ 2 and $a_{i j}>0$

$$
\lim _{t \rightarrow \infty}\left\|p_{i}(t)-p_{j}(t)\right\|=r_{i j}^{*}, \quad \forall j \in \mathscr{N}_{i},
$$

$3 \quad$ where $r_{i j}^{*}$ is the desired separation between robots $i$ and $j$, to be specified later.

$4 \quad$ Problem 2: (Distributed estimation of the desired separation): Develop a distributed estimation scheme 5 to estimate the desired separation $r_{i j}^{*}$ between robots $i$ and $j$ for any $i \neq j$ and $s_{i j}=1$ in the sense that $r_{i j}^{*}$ 6 captures the best tradeoff between communication quality and broadcasting coverage.

7 In essence, problem 1 is similar to the flocking problem but without the requirements of velocity 8 alignments and cohesion [8][9][11]. A common approach is to employ a potential field function that is 9 designed to be a convex function of distance, to be continuously differentiable (e.g., a $\sigma$-norm potential 10 function is proposed in [8] to ensure a smooth differentiation), and to attain the desired separation 11 as its unique minimum/maximum [3]. Despite of its effectiveness, a potential field function applied 12 to nonholonomic system often has singular configuration (i.e., local minimum), which occurs if the 13 nonholonomic systems are initially coincide with each other $\left(r_{i j} \triangleq\left\|p_{i}-p_{j}\right\|=0, \forall i \neq j\right)$ or $v_{i}=0$ 14 for some $i$.

Inspired by the potential function approach, we propose the following distance based formation con-

17 Specifically, for robot $i$, the controls are chosen as

18

$$
\begin{aligned}
v_{i} & =\mu \sum_{j \in \mathscr{N}_{i}} a_{i j}\left(r_{i j}-r_{i j}^{*}\right) \cos \left(\psi_{i j}-\theta_{i}\right), \\
\omega_{i} & =\mu^{\prime} \sum_{j \in \mathscr{N}_{i}} a_{i j}\left(r_{i j}-r_{i j}^{*}\right) \sin \left(\psi_{i j}-\theta_{i}\right),
\end{aligned}
$$

where $\mu^{\prime} \geq \mu>0$ are control gains to be selected, $\psi_{i j}$ is the line-of-sight (i.e., LOS) angle between robots $i$ and $j$ with $\psi_{i j}=\operatorname{atan} 2\left(y_{j}-y_{i}, x_{j}-x_{i}\right)$ and $\psi_{j i}=\pi+\psi_{i j}$. Note that $\left(\psi_{i j}-\theta_{i}\right)$ is the angle from the velocity vector of the $i$ th vehicle to the line segment connecting the centers of the $i$ th and $j$ th vehicles.

It should be noted that control (6) can be expressed as

$$
\begin{aligned}
v_{i}= & \mu \sum_{j \in \mathscr{N}_{i}} a_{i j}\left\{\left[\left(x_{j}+\frac{r_{i j}^{*}}{2} \cos \psi_{j i}\right)-\left(x_{i}+\frac{r_{i j}^{*}}{2} \cos \psi_{i j}\right)\right] \cos \theta_{i}\right. \\
& \left.+\left[\left(y_{j}+\frac{r_{i j}^{*}}{2} \sin \psi_{j i}\right)-\left(y_{i}+\frac{r_{i j}^{*}}{2} \sin \psi_{i j}\right)\right] \sin \theta_{i}\right\} .
\end{aligned}
$$

It follows from (8) that the proposed scheme converts the distance based formation problem to a rendezvous problem of a group of circular objects with a radius of $\frac{r_{i j}^{*}}{2}$. Moreover, it is clear that term $\left(r_{i j}-r_{i j}^{*}\right)$ in control (6) behaves like a time varying gain whose sign is determined dynamically by comparing the current and desired separations. Furthermore, the term acts as an attractive force if $r_{i j}>r_{i j}^{*}$, and robot $i$ 
moves to reduce separation $r_{i j}$ if $s_{i j}=1$; the term becomes repulsive once $r_{i j}<r_{i j}^{*}$, and it drives robot $i$ 2 away from vehicle $j$. Intuitively, the desired separation $r_{i j}^{*}$ for all $j \in \mathscr{N}_{i}$ is ensured distributively provided 3 that the network is connected. Moreover, using $\psi_{i j}$ in formation control (6) and (7) is advantageous in 4 the sense that it effectively eliminates the aforementioned singular configuration, since $\sin \psi_{i j}$ and $\cos \psi_{i j}$ 5 cannot be zero simultaneously. Nonetheless, it should be pointed out that local minimum problem may 6 still persist due to communication shadowing, where the short-term average received power is not strictly inversely proportional to distance. For the sake of brevity, communication shadowing will not be addressed s further in this paper. In addition, $\omega_{i}$ is designed to be perpendicular to $v_{i}$ (i.e., $\omega_{i} \perp v_{i}$ ) such that both inputs constitute a moving frame and, as a result, the resulted trajectory is smooth. Performance of the proposed inputs (6) and (7) is summarized into the following theorem. Choice (9) is not unique, and all choices of $a_{i j}$ are acceptable as long as $a_{i j^{*}}$ are row dominant.

Theorem 1: Consider a group of $n$ robots described by (5) and graph $\mathscr{G}$ satisfying assumptions 1. 13 Then, for any choice of $r_{i j}^{*}$ that ensures communication connectivity (for $r_{i j}^{*}<r_{i j}$ ), problem 1 is solved 14 in an asymptotical manner and graph $\mathscr{G}$ stays connected for any $t>t_{0}$ provided that $v_{i}$ and $\omega_{i}$ are 15 chosen according to (6) and (7), respectively, and that weightings $a_{i j}$ are chosen according to (1) and 16 the following:

$$
a_{i j}=\left\{\begin{array}{ll}
1 / 2 & \text { if } i \neq j, j=j^{*}, \text { where } j^{*} \in \mathscr{N}_{i} \text { is chosen such that }\left|r_{i j^{*}}-r_{i j^{*}}^{*}\right|=\max _{k \in \mathscr{N}_{i}}\left|r_{i k}-r_{i k}^{*}\right| \\
\frac{1}{2\left(n^{*}-1\right)} & \text { if } i \neq j, j \neq j^{*}, \text { and } j \in \mathscr{N}_{i}
\end{array},\right.
$$

17 where $n^{*}$ is the cardinality of neighboring set $\mathscr{N}_{i}$.

18 Proof: Consider the following Lyapunov function candidate:

$$
V=\frac{1}{2 \mu} \sum_{i, j=1}^{n}\left(r_{i j}-r_{i j}^{*}\right)^{2}
$$

19 It follows that time derivative of $V$ along (5) is

$$
\dot{V}=\frac{1}{\mu} \sum_{i, j=1}^{n}\left(1-\frac{r_{i j}^{*}}{r_{i j}}\right)\left[\left(x_{i}-x_{j}\right)\left(\dot{x}_{i}-\dot{x}_{j}\right)+\left(y_{i}-y_{j}\right)\left(\dot{y}_{i}-\dot{y}_{j}\right)\right] .
$$

20 Substituting (6) into (10) and using the facts that

$$
x_{j}-x_{i}=r_{i j} \cos \psi_{i j}, \quad y_{j}-y_{i}=r_{i j} \sin \psi_{i j}, \quad \text { and } a_{i j}=a_{j i},
$$

21 and that

$$
\begin{aligned}
\frac{1}{\mu} \frac{d\left(r_{i j}-r_{i j}^{*}\right)}{d t}= & -a_{i j}\left(r_{i j}-r_{i j}^{*}\right)\left[\cos ^{2}\left(\theta_{j}-\psi_{i j}\right)+\cos ^{2}\left(\theta_{i}-\psi_{i j}\right)\right] \\
& +\left[\sum_{k \neq j, k \in \mathscr{N}_{i}} a_{i k}\left(r_{i k}-r_{i k}^{*}\right) \cos \left(\theta_{i}-\psi_{i k}\right) \cos \left(\theta_{i}-\psi_{i j}\right)\right. \\
& \left.-\sum_{\ell \neq i, \ell \in \mathscr{N}_{j}} a_{j \ell}\left(r_{j \ell}-r_{j \ell}^{*}\right) \cos \left(\theta_{j}-\psi_{j \ell}\right) \cos \left(\theta_{j}-\psi_{i j}\right)\right] .
\end{aligned}
$$


1 Therefore, after rearranging all the terms in (11), we have

$$
\dot{V}=-\sum_{i, j=1}^{n} a_{i j}\left(r_{i j}-r_{i j}^{*}\right)^{2}\left[\cos ^{2}\left(\theta_{j}-\psi_{i j}\right)+\cos ^{2}\left(\theta_{i}-\psi_{i j}\right)\right] .
$$

2 Therefore, $\dot{V} \leq 0$ is ensured, and since there exists at least one gain $a_{i j}>0$ should $\mathscr{G}$ be connected, 3 therefore, by invoking LaSalle principle [24], it is clear that $\left(\theta_{i}-\psi_{i j}\right)=\pi / 2$ is not an equilibrium and 4 hence $\dot{V}<0$ is ensured for any $r_{i j} \neq r_{i j}^{*}$. As such, the desired separation can be achieved asymptotically.

5 To study the network connectivity, we propose the following Lyapunov function candidate,

$$
V^{\prime}=\frac{1}{2 \mu}\left(r_{i^{\star} j^{\star}}-r_{i^{\star} j^{\star}}^{*}\right)^{2},
$$

6 where systems $i^{\star}$ and $j^{\star}$ satisfy

$$
\left(i^{\star}, j^{\star}\right)=\left\{(i, j): \max _{i, j}\left|r_{i j}-r_{i j}^{*}\right|\right\}
$$

7 Analogously, we have

$$
\begin{aligned}
\dot{V}^{\prime}= & -a_{i^{\star} j^{\star}}\left(r_{i^{\star} j^{\star}}-r_{i^{\star} j^{\star}}^{*}\right)^{2}\left[\cos ^{2}\left(\theta_{j^{\star}}-\psi_{i^{\star} j^{\star}}\right)+\cos ^{2}\left(\theta_{i^{\star}}-\psi_{i^{\star} j^{\star}}\right)\right] \\
& +\left(r_{i^{\star} j^{\star}}-r_{i^{\star} j^{\star}}^{*}\right)\left[\sum_{k \neq j^{\star}, k \in \mathscr{N}_{i}} a_{i^{\star} k}\left(r_{i^{\star} k}-r_{i^{\star} k}^{*}\right) \cos \left(\theta_{i^{\star}}-\psi_{i^{\star} k}\right) \cos \left(\theta_{i^{\star}}-\psi_{i^{\star} j^{\star}}\right)\right. \\
& \left.-\sum_{\ell \neq i^{\star}, \ell \in \mathscr{N}_{j}} a_{j^{\star} \ell}\left(r_{j^{\star} \ell}-r_{j^{\star} \ell}^{*}\right) \cos \left(\theta_{j^{\star}}-\psi_{j^{\star} \ell}\right) \cos \left(\theta_{j^{\star}}-\psi_{i^{\star} j^{\star}}\right)\right] .
\end{aligned}
$$

8 It follows from (9) that $a_{i^{\star} j^{\star}}$ is the dominant gain and $a_{i^{\star} j^{\star}}=\sum_{k=1, k \neq j^{\star}} a_{i k}$. Hence, $\dot{V}^{\prime} \leq 0$ is ensured if

$$
\left|\cos \left(\theta_{j^{\star}}-\psi_{i^{\star} j^{\star}}\right)\right| \geq\left|\cos \left(\theta_{j^{\star}}-\psi_{i^{\star} k}\right)\right| \text {. }
$$

9 Note that condition (14) may not hold all the time, e.g., the worst case is $\left(\theta_{j^{\star}}-\psi_{i^{\star} j^{\star}}\right)=\pi / 2$. Nonetheless, 10 it follows from (7) that

$$
\dot{\theta}_{j^{\star}}=\mu^{\prime} \sum_{k=1}^{n} a_{j^{\star} k}\left(r_{j^{\star} k}-r_{j^{\star} k}^{*}\right) \sin \left(\psi_{j^{\star} k}-\theta_{j^{\star}}\right),
$$

11 that its right hand side is dominated by the term of $\left(r_{j^{\star} i^{\star}}-r_{j^{\star} i^{\star}}\right) \sin \left(\psi_{j^{\star} i^{\star}}-\theta_{j^{\star}}\right)$ and hence

$$
\theta_{j^{\star}} \rightarrow \psi_{i^{\star} j^{\star}}
$$

12 By simply increasing $\mu^{\prime}$, condition (14) can be made valid very quickly. Combining the above arguments 13 completes the proof.

14 Remark 1*: It is well known that either a time-varying control or a discontinuous control is needed 15 to ensure asymptotic stability for nonholonomic system (5). Controls (6) and (7) are discontinuous in 16 nature due to the state-dependent gains $a_{i j}$ defined by (9). Equation (12) reveals that the configuration

\footnotetext{
*The authors owe this observation to an anonymous reviewer.
} 


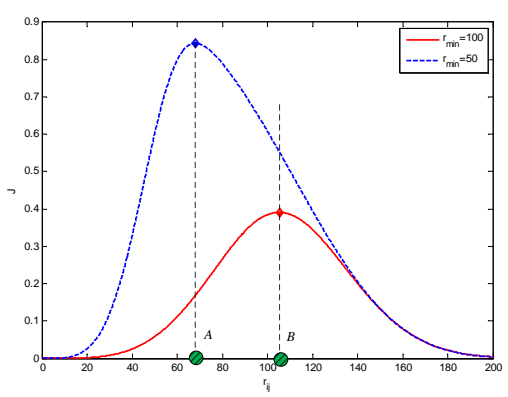

Fig. 3: Performance index $J$ with $r_{x}=150, v=4$

$1 \theta_{i}=\theta_{j}=\psi_{i j}+\pi / 2$ renders $v_{i}=0$ for vehicle (5) under control (6). Whenever this occurs, the choices of $2 a_{i j}$ and control (7) ensure that $\theta_{i}$ and $\theta_{j}$ are steered away from $\psi_{i j}+\pi / 2$ and toward $\psi_{i j}$, as evidenced 3 by (15). Accordingly, $v_{i}=0$ can only hold instantaneously unless $r_{i j}=r_{i j}^{*}$. In essence, with the inclusion 4 of line-of-sight angle (i.e., $\psi_{i j}$ ), controls (6) and (7) are reduced to control a projection of the heading 5 angle $\theta_{i}$, instead of dealing with nonholonomic restrictions directly. As a result, the proposed controller 6 is resilient to nonholonomic constraints and the resulted trajectory is feasible.

7 It follows from theorem 1 that, should $r_{i j}^{*}$ be known or determined for any $j \in \mathscr{N}_{i}$, the proposed formation 8 control scheme ensures an asymptotic coverage with the desired separation. Hence, it is imperative to 9 formulate a performance index, whose solution is $r_{i j}^{*}$, as the goal of problem 2 . To this end, the following 10 index is proposed, whose effectiveness has been verified in [1]:

$$
J\left(r_{i j}\right)=\left[1-\exp \left(-\left(\frac{r_{i j}}{r_{\min }}\right)^{v}\right)\right] \cdot \exp \left(-\left(\frac{r_{i j}}{r_{x}}\right)^{v}\right) \cdot \exp \left(-\left(2^{\delta}-1\right) \frac{\sigma^{2}}{P_{0}}\left(\frac{r_{i j}}{r_{0}}\right)^{v}\right), \quad \forall j \in \mathscr{N}_{i},
$$

11 where $r_{\min }$ is the minimum spacing preferred, $r_{x}$ is the (maximum) spacing that renders the worst tolerable 12 outage probability, and $v$ is a tuning parameter.

Note that the choice of performance index could vary due to different design objectives, provided that 14 the resulted index is a convex function of the optimal solution $r_{i j}^{*}$ and has a unique minimum/maximum. 15 Moreover, it is worth stressing that the exact value of $J\left(r_{i j}\right)$ is not known locally since outage probability 16 can only be measured by estimating/measuring the data packets received [15]. Considering the cases 17 in figure 1, performance index $J\left(r_{i j}\right)$ and its maximum corresponding to $\left\{r_{x}=150, r_{\min }=50\right\}$ and ${ }_{18}\left\{r_{x}=150, r_{\min }=100\right\}$ are shown in figure 3 , and their corresponding outage probabilities are the same 19 as $A$ and $B$ in figure 1 . It is clear the case with $r_{\min }=50$ corresponds to a better quality of service (i.e., outage probability 9\%), while the case with $r_{\min }=100$ achieves better network coverage (i.e., $r_{i j}^{*}=105$ ).

21 Accordingly, cooperative control (6) and (7) shall be integrated with and assisted by an algorithm of searching for maximum $r_{i j}^{*}$ with respect to unknown $J\left(r_{i j}\right)$, their combination drives the separation of robots to the desired value of $r_{i j}^{*}$. Accordingly, the tradeoff between network coverage and communication 
quality is ensured.

\section{DistRIBUTED EXTREMUM SEEKING FOR FORMATION CONTROL}

In this section, problem 2 will be solved by using the approach of distributed extremum seeking, and, compared to the classic extremum seeking algorithm, the proposed scheme can perform individual estimation with acceptable accuracy, as well as reach a consensus between any pair of connected robots, at the aforementioned topological requirement. In what follows, consensus is said to be reached between $x$ and $y$ if $|x-y| \leq \varepsilon$, with $\varepsilon>0$ being a predefined constant, and in this case we denote $x \vec{\sim} y$ or $y \vec{\sim} x$. Hence, the intuition of distributed extremum seeking is that, shall there exist an undirected link between robots $i$ and $j$ (i.e., $s_{i j}=s_{j i}=1$ ), consensus among $\hat{r}_{i j} \vec{\sim} r_{i j}^{*}$ and $\hat{r}_{i j} \vec{\sim} \hat{r}_{j i}$ should be ensured asymptotically.

As has been discussed, neither the exact value of outage probability (3) nor its gradient is known locally, and the only information available about communication quality is the measurement of outage probability. As such, no analytical solution is available nor it is possible to derive the maximum of performance index $J\left(r_{i j}\right)$, which calls for a model-free scheme to search for maximum of $J\left(r_{i j}^{*}\right)$. Therefore, extremum seeking control would be a natural framework for the underlying problem [25]. However, it should be pointed out that extremum seeking scheme must be enhanced when applied to multi-agent formation control problems, where not only the desired separation should be estimated accurately, but more importantly, formation stability among any pair of connected systems should also be ensured. In other words, consensus between estimates should be guaranteed in order to render a stable formation.

In addition, extremum seeking shall be implemented using only existing communication channels such that the desired separation $r_{i j}^{*}$ can be determined distributively for all $j \in \mathscr{N}_{i}$. As shown in figure 3 , there exists $r_{i j}=r_{i j}^{*}$ such that $J\left(r_{i j}^{*}\right)=\max _{j \in \mathscr{N}_{i}} J\left(r_{i j}\right)$, which implies

$$
J^{\prime}\left(r_{i j}^{*}\right)=0, \quad J^{\prime \prime}\left(r_{i j}^{*}\right)<0 .
$$

In order to estimate $r_{i j}^{*}$ and ensure a consensus among estimates, we propose the following distributed extremum seeking scheme, as illustrated in figure 4:

$$
\left\{\begin{array}{l}
\dot{\hat{r}}_{i j}=\xi_{i j}+\kappa s_{i j}\left(\hat{r}_{j i}-\hat{r}_{i j}\right) \\
\dot{\xi}_{i j}=\beta_{l}\left\{-\xi_{i j}+\left[J\left(\hat{r}_{i j}+a \sin \beta_{0} t\right)-\eta_{i j}\right] a \sin \beta_{0} t+\kappa s_{i j}\left(\hat{r}_{i j}-\hat{r}_{j i}\right)\right\} \\
\dot{\eta}_{i j}=\beta_{h}\left[-\eta_{i j}+J\left(\hat{r}_{i j}+a \sin \beta_{0} t\right)\right]
\end{array}\right.
$$

where $\hat{r}_{i j}$ is the current estimate of $r_{i j}^{*}, s_{i j}$ is the $(i, j)$-th entry of matrix $S(t)$ as defined in (2), $\xi_{i j}$ and $\eta_{i j}$ are intermediate variables used in extremum seeking, and $a, \beta_{0}, \kappa$ are the gains to be specified.

It follows from (18) that the proposed distributed scheme reduces to the existing individual extremum seeking scheme if $s_{i j}=0$, in which case extremum seeking can still be performed, but there is no 


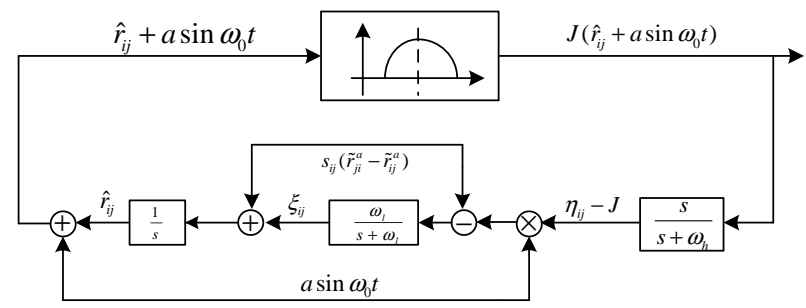

Fig. 4: Diagram of extremum seeking

1 information exchange with any of the neighbors and any inconsistency between $\hat{r}_{i j}$ and $\hat{r}_{j i}$ (i.e., $\forall i \neq j$ ) 2 would be accumulated and may prevent a stable formation. In the event of $s_{i j}(t)=1$, a cooperative control 3 algorithm is integrated with extremum seeking control in the sense that robots $i$ and $j$ can exchange 4 information of current estimation (i.e., $\hat{r}_{i j}$ ), which leads to a consensus of their estimates. Performance of 5 the proposed scheme is summarized into the following theorem.

6 Theorem 2: Consider a group of n robots described by (5), and each of the robots performs their formation 7 control according to (6) and (7). Let $J\left(r_{i j}\right)$ be the performance index defined in (16), and it is assumed 8 that the value of $J\left(r_{i j}\right)$ can be measured and reaches its maximum at $r_{i j}^{*}$. Then, under assumption 1, the 9 proposed distributive extremum seeking scheme (18) solves problem 2 in the sense that, as $t \rightarrow \infty$

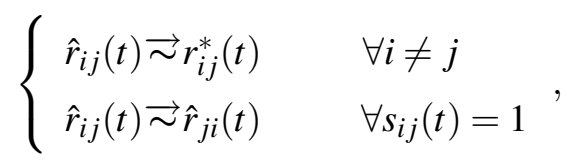

10 provided $\beta_{0} \gg 1, \beta_{h}>\beta_{l}>0, \kappa=\frac{\beta_{l}^{2}}{4}$ and $0<a \ll 1$ being a sufficiently small constant. Specifically, 11 estimation error (i.e., $\left.\left(\hat{r}_{i j}-r_{i j}^{*}\right)\right)$ can be ensured to a neighborhood of size $O\left(\frac{1}{\beta_{0}}+a^{3}\right)$ around the origin. 12 Proof: The first part of the proof is to show that $r_{i j}^{*}$ can be individually estimated. Define the estimation 13 errors as

$$
\tilde{r}_{i j}=\hat{r}_{i j}-r_{i j}^{*}, \quad \tilde{\eta}_{i j}=\eta_{i j}-J\left(r_{i j}^{*}\right) .
$$

${ }_{14}$ Then, averaging the trajectory of system (18) over the period of $2 \pi$ in the time scale of $\tau=\beta_{0} t$ yields

$$
\frac{d}{d \tau}\left[\begin{array}{c}
\tilde{r}_{i j}^{a} \\
\xi_{i j}^{a} \\
\tilde{\eta}_{i j}^{a}
\end{array}\right]=\frac{1}{\beta_{0}}\left[\begin{array}{c}
\xi_{i j}^{a}+\kappa s_{i j}\left(\tilde{r}_{j i}^{a}-\tilde{r}_{i j}^{a}\right) \\
-\beta_{l} \xi_{i j}^{a}+\frac{\beta_{l}}{2 \pi} a \int_{0}^{2 \pi} \rho\left(\tilde{r}_{i j}^{a}+a \sin \vartheta\right) \sin \vartheta d \vartheta+\kappa \beta_{l} s_{i j}\left(\tilde{r}_{i j}^{a}-\tilde{r}_{j i}^{a}\right) \\
-\beta_{h} \tilde{\eta}_{i j}^{a}+\frac{\beta_{h}}{2 \pi} \int_{0}^{2 \pi} \rho\left(\tilde{r}_{i j}^{a}+a \sin \vartheta\right) d \vartheta
\end{array}\right],
$$

where $\rho(x)=J\left(r_{i j}^{*}+x\right)-J\left(r_{i j}^{*}\right)$, and variables with superscript $a$ denote the state variables of the averaged system.

It follows from (17) that,

$$
\rho(0)=0, \rho^{\prime}(0)=J^{\prime}\left(r_{i j}^{*}\right)=0, \rho^{\prime \prime}(0)=J^{\prime \prime}\left(r_{i j}^{*}\right)<0,
$$


1 where $\rho^{\prime}(x)=\frac{\partial \rho(x)}{\partial x}, \rho^{\prime \prime}(x)$ and $\rho^{\prime \prime \prime}(x)$ can be defined accordingly. Then, the average system equilibrium $2\left(\tilde{r}_{i j}^{a, e}, \xi_{i j}^{a, e}, \tilde{\eta}_{i j}^{a, e}\right)$ should satisfy the following relations:

$$
\xi_{i j}^{a, e}=-\kappa s_{i j}\left(\tilde{r}_{j i}^{a, e}-\tilde{r}_{i j}^{a, e}\right), \quad \int_{0}^{2 \pi} \rho\left(\tilde{r}_{i j}^{a, e}+a \sin \vartheta\right) \sin \vartheta d \vartheta=0, \quad \tilde{\eta}_{i j}^{a, e}=\frac{1}{2 \pi} \int_{0}^{2 \pi} \rho\left(\tilde{r}_{i j}^{a, e}+a \sin \vartheta\right) d \vartheta
$$

$3 \quad$ Assume that $\tilde{r}_{i j}^{a, e}$ be parameterized as

$$
\tilde{r}_{i j}^{a, e}=b_{i j}^{(1)} a+b_{i j}^{(2)} a^{2}+O\left(a^{3}\right),
$$

4 where $b_{i j}^{(1)}$ and $b_{i j}^{(2)}$ are the constants to be determined. Substituting (23) into (22), we have $b_{i j}^{(1)}=0$, ${ }_{5} \quad b_{i j}^{(2)}=-\frac{\rho^{\prime \prime \prime}(0)}{8 \rho^{\prime \prime}(0)}$ and $\xi_{i j}^{a, e} \leq O\left(a^{3}\right)$. After several algebraic manipulations, equilibrium $\left(\tilde{r}_{i j}^{a, e}, \xi_{i j}^{a, e}, \tilde{\eta}_{i j}^{a, e}\right)$ of 6 system (21) is found to satisfy:

$$
\left|\begin{array}{c}
\tilde{r}_{i j}^{a, e} \\
\xi_{i j}^{a, e} \\
\tilde{\eta}_{i j}^{a, e}
\end{array}\right| \approx\left[\begin{array}{c}
-\frac{\rho^{\prime \prime \prime}(0)}{8 \rho^{\prime \prime}(0)} a^{2}+O\left(a^{3}\right) \\
O\left(a^{3}\right) \\
\frac{\rho^{\prime \prime}(0)}{4} a^{2}+O\left(a^{3}\right)
\end{array}\right]
$$

7 This implies that estimation errors converges as $\tilde{r}_{i j}^{a, e}, \tilde{\eta}_{i j}^{a, e} \underset{\sim 0}{ }$ for a sufficiently small choice of $a$. In 8 addition, it has been established in [25] that the Jacobian of average system (21) is Hurwitz if and only if 9 $\int_{0}^{2 \pi} \rho\left(\tilde{r}_{i j}^{a}+a \sin \vartheta\right) \sin \vartheta d \vartheta<0$, and consequently the unique exponentially stable solution $\left(\tilde{r}_{i j}^{2 \pi}, \xi_{i j}^{2 \pi}, \tilde{\eta}_{i j}^{2 \pi}\right)$ 10 satisfies the relationship

$$
\left|\left[\begin{array}{c}
\tilde{r}_{i j}^{2 \pi}+\frac{\rho^{\prime \prime \prime}(0)}{8 \rho^{\prime \prime}(0)} a^{2} \\
\xi_{i j}^{2 \pi} \\
\tilde{\eta}_{i j}^{2 \pi}-\frac{\rho^{\prime \prime}(0)}{4} a^{2}
\end{array}\right]\right| \approx O\left(\frac{1}{\beta_{0}}+a^{3}\right),
$$

11 which implies all solutions $\left(\tilde{r}_{i j}, \xi_{i j}, \tilde{\eta}_{i j}\right)$ converge to a size- $O\left(\frac{1}{\beta_{0}}+a^{3}\right)$ neighborhood around the origin. 12 That is, the estimation errors can be made arbitrarily small provided that $\beta_{0}$ is sufficiently large and $a$ is 13 sufficiently small. That is, $\hat{r}_{i j} \vec{\sim} r_{i j}^{*}$ is ensured as $t \rightarrow \infty$.

The next step is to prove that consensus between estimates for any pair of connected robots can be motion dynamics of robot $i$ using the same time scale as that of (21) yields

$$
\left\{\begin{aligned}
\dot{x}_{i}^{a} & =\frac{\mu}{\beta_{0}} \sum_{j \in \mathscr{N}_{i}} a_{i j}\left(r_{i j}^{a}-r_{i j}^{*}-\tilde{r}_{i j}^{a}\right)\left[\cos \psi_{i j}^{a} \cos ^{2} \theta_{i}^{a}+\sin \psi_{i j}^{a} \cos \theta_{i}^{a} \sin \theta_{i}^{a}\right], \\
\dot{y}_{i}^{a} & =\frac{\mu}{\beta_{0}} \sum_{j \in \mathscr{N}_{i}} a_{i j}\left(r_{i j}^{a}-r_{i j}^{*}-\tilde{r}_{i j}^{a}\right)\left[\cos \psi_{i j}^{a} \sin \theta_{i}^{a} \cos \theta_{i}^{a}+\sin \psi_{i j}^{a} \sin ^{2} \theta_{i}^{a}\right] .
\end{aligned}\right.
$$

17 It is apparent that, although the above averaged and networked control system is coupled to extremum seeking system (21) at the time scale of $\tau=\beta_{0} t$, it is approximately equivalent to system (5) under inputs (6) and (7) since estimation error (i.e., $\tilde{r}_{i j}^{a}$ ) is sufficiently small. In other words, large value of perturbation frequency $\beta_{0}$ have insignificant impact on networked control system, and the convergence and control can 
1 be achieved by making proper choices of design parameters, as to be specified later. Consequently, the 2 averaged closed-loop control and estimation system at robot $i$ becomes

$$
\frac{d}{d \tau}\left[\begin{array}{c}
x_{i}^{a} \\
y_{i}^{a} \\
\tilde{r}_{i j}^{a} \\
\xi_{i j}^{a} \\
\tilde{\eta}_{i j}^{a}
\end{array}\right]=\frac{1}{\beta_{0}}\left[\begin{array}{c}
\mu \sum_{j \in \mathscr{N}_{i}} a_{i j}\left(r_{i j}^{a}-r_{i j}^{*}-\tilde{r}_{i j}^{a}\right)\left[\cos \psi_{i j}^{a} \cos ^{2} \theta_{i}^{a}+\sin \psi_{i j}^{a} \cos \theta_{i}^{a} \sin \theta_{i}^{a}\right] \\
\mu \sum_{j \in \mathscr{N}_{i}} a_{i j}\left(r_{i j}^{a}-r_{i j}^{*}-\tilde{r}_{i j}^{a}\right)\left[\cos \psi_{i j}^{a} \sin \theta_{i}^{a} \cos \theta_{i}^{a}+\sin \psi_{i j} \sin ^{2} \theta_{i}^{a}\right] \\
\beta_{l}\left[-\xi_{i j}^{a}+\frac{a}{2 \pi} \int_{0}^{2 \pi} \rho\left(\tilde{r}_{i j}^{a}+a \sin \vartheta\right) \sin \vartheta d \vartheta+\kappa s_{i j}\left(\tilde{r}_{i j}^{a}-\tilde{r}_{j i}^{a}-\tilde{r}_{i j}^{a}\right)\right. \\
\beta_{h}\left[-\tilde{\eta}_{i j}^{a}+\frac{1}{2 \pi} \int_{0}^{2 \pi} \rho\left(\tilde{r}_{i j}^{a}+a \sin \vartheta\right) d \vartheta\right] .
\end{array}\right] .
$$

3 To study the performance of the integrated cooperative estimation/control and extremum seeking system 4 of (27), we propose the following Lyapunov function candidate,

$$
V_{c}=\frac{\beta_{0}}{2} \sum_{i, j=1}^{n} s_{i j}(t)\left[\frac{\beta_{l}^{2}}{2}\left(\tilde{r}_{i j}^{a}-\tilde{r}_{j i}^{a}\right)^{2}+\frac{1}{\beta_{l}}\left(\xi_{i j}^{a}-\xi_{j i}^{a}\right)^{2}+\frac{a}{\beta_{h}}\left(\tilde{\eta}_{i j}^{a}-\tilde{\eta}_{j i}^{a}\right)^{2}\right] .
$$

5 It is clear that $V_{c}$ is positive semi-definite and radially unbounded, and $s_{i j}(t)$ is included to deterministically 6 capture the contribution of connectivity between robots $i$ and $j$. Taking time derivative of $V_{c}$ along the 7 trajectory of (27) yields

$$
\dot{V}_{c}=\sum_{i, j=1}^{n} s_{i j}(t)\left\{\frac{\beta_{l}^{2}}{2}\left(\tilde{r}_{i j}^{a}-\tilde{r}_{j i}^{a}\right)\left(\dot{\tilde{r}}_{i j}^{a}-\dot{\tilde{r}}_{j i}^{a}\right)+\left(\xi_{i j}^{a}-\xi_{j i}^{a}\right)\left(\dot{\xi}_{i j}^{a}-\dot{\xi}_{j i}^{a}\right)+\frac{a}{\beta_{h}}\left(\tilde{\eta}_{i j}^{a}-\tilde{\eta}_{j i}^{a}\right)\left(\dot{\tilde{\eta}}_{i j}^{a}-\dot{\tilde{\eta}}_{j i}^{a}\right)\right\} .
$$

8 Consequently,

$$
\begin{aligned}
\dot{V}_{c}= & \sum_{i, j=1}^{n} s_{i j}(t)\left\{-\frac{\kappa \beta_{l}^{2}}{2}\left(s_{i j}+s_{j i}\right)\left(\tilde{r}_{i j}^{a}-\tilde{r}_{j i}^{a}\right)^{2}-\left(\xi_{i j}^{a}-\xi_{j i}^{a}\right)^{2}-a\left(\tilde{\eta}_{i j}^{a}-\tilde{\eta}_{j i}^{a}\right)^{2}\right. \\
& +\left[\kappa\left(s_{i j}+s_{j i}\right)+\beta_{l}^{2} / 2\right]\left(\xi_{i j}^{a}-\xi_{j i}^{a}\right)\left(\tilde{r}_{i j}^{a}-\tilde{r}_{j i}^{a}\right) \\
& +\frac{a}{2 \pi}\left(\xi_{i j}^{a}-\xi_{j i}^{a}\right)\left(\int_{0}^{2 \pi} \rho\left(\tilde{r}_{i j}^{a}+a \sin \vartheta\right) \sin \vartheta d \vartheta-\int_{0}^{2 \pi} \rho\left(\tilde{r}_{j i}^{a}+a \sin \vartheta\right) \sin \vartheta d \vartheta\right) \\
& \left.+\frac{a}{2 \pi}\left(\tilde{\eta}_{i j}^{a}-\tilde{\eta}_{j i}^{a}\right)\left(\int_{0}^{2 \pi} \rho\left(\tilde{r}_{i j}^{a}+a \sin \vartheta\right) d \vartheta-\int_{0}^{2 \pi} \rho\left(\tilde{r}_{j i}^{a}+a \sin \vartheta\right) d \vartheta\right)\right\} .
\end{aligned}
$$

9 Recalling that, if $a$ is sufficiently small,

$$
\int_{0}^{2 \pi} \rho\left(\tilde{r}_{i j}^{a}+a \sin \vartheta\right) \sin \vartheta d \vartheta=0, \quad \int_{0}^{2 \pi} \rho\left(\tilde{r}_{i j}^{a}+a \sin \vartheta\right) d \vartheta=\frac{\rho^{\prime \prime}(0)}{4} a^{2}+O\left(a^{3}\right) .
$$

10 Hence, equation (30) becomes

$$
\begin{aligned}
\dot{V}_{c}= & \sum_{i, j=1}^{n} s_{i j}(t)\left\{-\frac{\kappa \beta_{l}^{2}}{2}\left(s_{i j}+s_{j i}\right)\left(\tilde{r}_{i j}^{a}-\tilde{r}_{j i}^{a}\right)^{2}-\left(\xi_{i j}^{a}-\xi_{j i}^{a}\right)^{2}-a\left(\tilde{\eta}_{i j}^{a}-\tilde{\eta}_{j i}^{a}\right)^{2}\right. \\
& \left.+\left[\kappa\left(s_{i j}+s_{j i}\right)+\frac{\beta_{l}^{2}}{2}\right]\left(\xi_{i j}^{a}-\xi_{j i}^{a}\right)\left(\tilde{r}_{i j}^{a}-\tilde{r}_{j i}^{a}\right)+\frac{a}{2 \pi}\left(\tilde{\eta}_{i j}^{a}-\tilde{\eta}_{j i}^{a}\right) O\left(a^{3}\right) .\right\}
\end{aligned}
$$




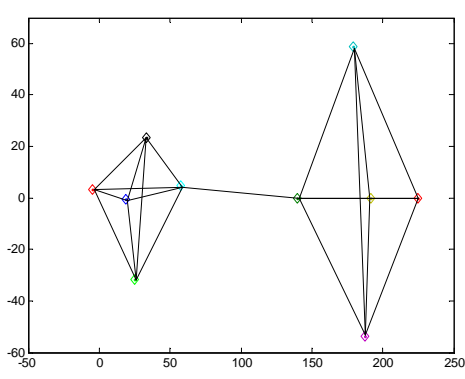

(a) $t=0 \sec$

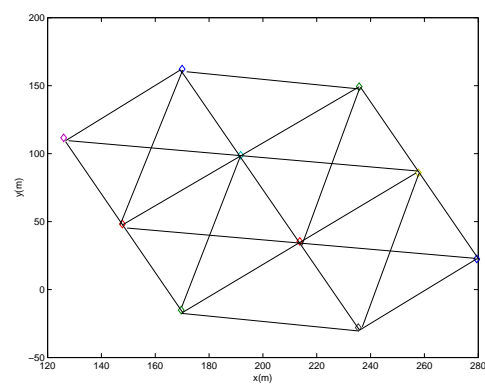

(b) $t=100 \sec$

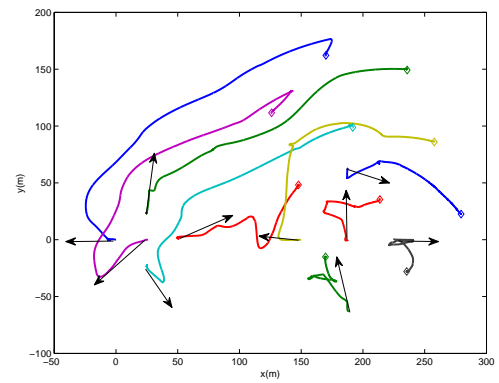

(c) Trajectories

Fig. 5: Evolution of formation movement and connectivity

1 It follows that, if graph is completely isolated (i.e., $s_{i j}=0$ for all $i \neq j$ ), $V_{c}=\dot{V}_{c}=0$, and no consensus 2 among estimates can be achieved. However, extremum seeking is individually performed under the 3 proposed scheme, and $r_{i j} \vec{\sim} r_{i j}^{*}$ is achieved as a result.

${ }_{4} \quad$ Shall robots $i$ and $j$ are connected (i.e., $s_{i j}=s_{j i}=1$ ), $a$ is sufficiently small and $\kappa=\frac{\beta_{l}^{2}}{4}$, equation (31) 5 becomes

$$
\dot{V}_{c}=-\sum_{i, j=1}^{n} s_{i j}(t)\{\underbrace{\left[\frac{\beta_{l}^{2}}{2}\left(\tilde{r}_{i j}^{a}-\tilde{r}_{j i}^{a}\right)-\left(\xi_{i j}^{a}-\xi_{j i}^{a}\right)\right]^{2}}_{\Lambda}+a\left(\tilde{\eta}_{i j}^{a}-\tilde{\eta}_{j i}^{a}\right)^{2}\} .
$$

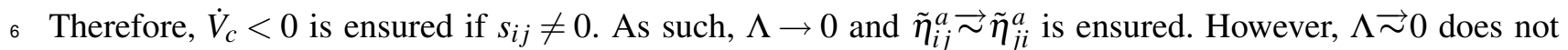
7 translate to $\tilde{r}_{i j}^{a} \rightleftharpoons \tilde{r}_{j i}^{a}$ and $\xi_{i j}^{a} \rightleftharpoons \xi_{j i}^{a}$, and in this worst case scenario, it could mean $\frac{\beta_{l}^{2}}{2}\left|\tilde{r}_{i j}^{a}-\tilde{r}_{j i}^{a}\right| \vec{\sim}\left|\xi_{i j}^{a}-\xi_{j i}^{a}\right|$. In 8 this event, $\left|\tilde{r}_{i j}^{a}-\tilde{r}_{j i}^{a}\right| \vec{\sim} \frac{2}{\beta_{l}^{2}}\left|\xi_{i j}^{a}-\xi_{j i}^{a}\right|$. Therefore, $\tilde{r}_{i j}^{a} \vec{\sim} \tilde{r}_{j i}^{a}$ can be ensured for any $j \in \mathscr{N}_{i}$ provided that $\beta_{l}$ is 9 chosen to be a fairly large value. Since $r_{i j}^{*}=r_{j i}^{*}$ in practice and $\hat{r}_{i j} \vec{\sim} r_{i j}^{*}, \hat{r}_{j i} \vec{\sim} r_{j i}^{*}, \hat{r}_{i j}^{a} \vec{\sim} \hat{r}_{j i}^{a}$ is subsequently 10 concluded. That is, consensus among estimates can be ensured between robots $i$ and $j$ as long as $s_{i j}=1$. 11 This concludes the proof of theorem 2.

Remark 2: For implementation, both the cut-off frequencies of the low-pass or high-pass filters (i.e., $\beta_{l}$ and $\beta_{h}$ ) shall be smaller than perturbation signal $\beta_{0}$, and the overall estimation and control system shall 14 have two time scales: a faster time scale for formation control system (5), and a slower time scale for distributed extremum seeking scheme. In particular, the convergence rate of system (21) is proportional to $\frac{1}{\beta_{0}}$, while convergence of system (26) is in general determined by $\frac{\mu}{\beta_{0}}$. Hence, a simple way to achieve separation is letting $\mu>1$. In addition, it follows from (24) that $a^{3} \leq \varepsilon$ should also be enforced in order to ensure a consensus with acceptable accuracy. 


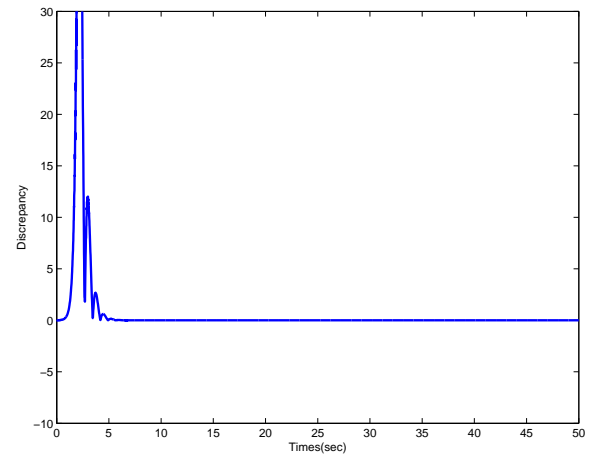

(a) $\sum_{i, j=1}^{n}\left|\hat{r}_{i j}-\hat{r}_{j i}\right|$

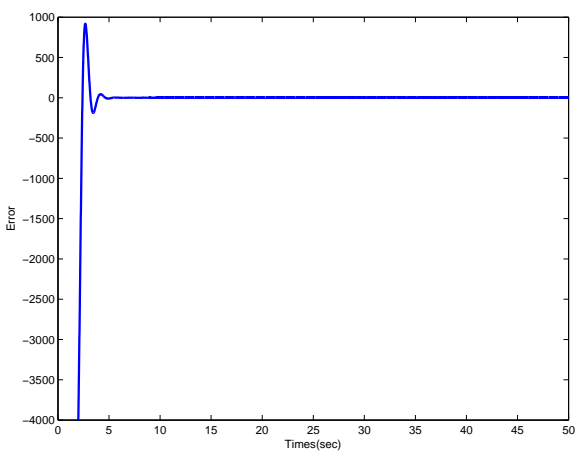

(b) $\sum_{i, j=1}^{n}\left|\hat{r}_{i j}-r_{i j}^{*}\right|$

Fig. 6: Performance of the proposed distributed extremum seeking

\section{Simulation RESULTS}

To illustrate performance of the proposed distributed extremum seeking scheme, consider a group of 310 robots whose initial conditions (in meters and radius) are: denoting $\mathbf{x}_{i}=\left[\begin{array}{ll}x_{i} & y_{i} \\ \omega_{i}\end{array}\right]^{T}$,

$$
\begin{gathered}
\mathbf{x}_{1}=\left[\begin{array}{lll}
0 & 0 & 0.26
\end{array}\right]^{T}, \mathbf{x}_{2}=\left[\begin{array}{lll}
25 & 25 & 0.17
\end{array}\right]^{T}, \mathbf{x}_{3}=\left[\begin{array}{lll}
50 & 0 & 0.35
\end{array}\right]^{T}, \mathbf{x}_{4}=\left[\begin{array}{lll}
25 & -25 & -0.17
\end{array}\right]^{T} \\
\mathbf{x}_{5}=\left[\begin{array}{lll}
25 & 0 & 0.52
\end{array}\right]^{T}, \mathbf{x}_{6}=\left[\begin{array}{lll}
150 & 0 & 0.17
\end{array}\right]^{T}, \mathbf{x}_{7}=\left[\begin{array}{lll}
225 & 0 & 1.05
\end{array}\right]^{T}, \mathbf{x}_{8}=\left[\begin{array}{lll}
188 & 63 & 0.79
\end{array}\right]^{T} \\
\mathbf{x}_{9}=\left[\begin{array}{lll}
188 & -63 & 0.56
\end{array}\right]^{T}, \mathbf{x}_{10}=\left[\begin{array}{lll}
188 & 0 & 0.87
\end{array}\right]^{T} .
\end{gathered}
$$

4 In simulations, cooperative control is implemented by utilizing only the motion information received from 5 neighboring robots ${ }^{\dagger}$ if $r_{i j} \leq r_{x}=150$ (its corresponding outage probability is shown at point $C$ as of figure

6 1). It is straightforward to see that the initial graph is barely connected and that the coverage is not well 7 spread, and that the communication quality among two separated groups is poor.

8 In the simulation, simulated value of performance index $J\left(r_{i j}\right)$ is calculated as

$$
\begin{aligned}
J\left(\hat{r}_{i j}+a \sin \beta_{0} t\right)= & {\left[1-\exp \left(-\left(\frac{\hat{r}_{i j}+a \sin \beta_{0} t}{r_{\min }}\right)^{v}\right)\right] \cdot \exp \left(-\left(\frac{\hat{r}_{i j}+a \sin \beta_{0} t}{r_{x}}\right)^{v}\right) } \\
& \times \exp \left(-\left(2^{\delta}-1\right) \frac{\sigma^{2}}{P_{0}}\left(\frac{\hat{r}_{i j}+a \sin \beta_{0} t}{r_{0}}\right)^{v}\right),
\end{aligned}
$$

9 with $r_{\min }=50$. The parameters characterizing communication quality in simulations are assumed to be the same for all the communication channels, and their values used in the formation control and extremum seeking are chosen as

$$
\mu=1, \beta_{0}=100, \mu=10, \beta_{l}=4, \beta_{h}=25, a=0.2, \kappa=4, \zeta=1, v=2, v=3, \varepsilon=0.01 .
$$

12 Evolution of the mobile communication network is shown in figure 5 (in which the presence of a 13 link between any pair of robots means their communication channels are considered to be of good 
quality). Clearly, the final configuration provides much improved performance, the separations among the neighboring robots automatically converge to the optimal value of $r_{i j}^{*}=68$, and the trajectories are 3 smooth over time. Moreover, performance of the proposed distributed extremum seeking is quantitatively 4 illustrated in figure 6. Specifically, figure 6a shows that consensus among estimates between any pair of 5 connected robots can be achieved promptly, while figure $6 \mathrm{~b}$ verifies that the desired separation can be

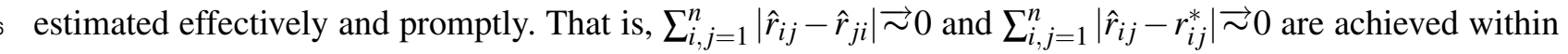
10 seconds.

\section{CONCLUSION}

In this paper, the integrated control and communication problem is investigated for a group of dynamical systems under undirected graph. The proposed control/estimation/optimization solution is based on two novel ideas: a performance metric capturing the trade off between communication quality and network coverage, and a distributed extremum seeking algorithm that also incorporates cooperative control.

In addition, the proposed cooperative formation control scheme uses only local measurements and line-of-sight (i.e., LOS) bearing and separations to avoid any local minimal problem and to achieve the desired separation. The proposed control/estimation algorithm renders the optimal separation for the defined performance index and subsequently formation control, but the final configuration may not be optimal or unique (i.e., the final configuration is determined by both the initial condition and the graph) because it uses only the measurement of relative distance/LOS. A distributive estimation/control scheme that ensures the optimal final configuration should be of interest for the future development on this topic.

\section{ACKNOWLEDGEMENT}

The authors would like to thank the associate editor and all anonymous reviewers for their constructive comments that improved the quality of this paper.

[1] C. Li, Z. Qu, and M. A. Ingram, "Distributed extremum seeking and cooperative control for mobile communication," in IEEE Conference on Decision and Control and European Control Conference (CDC-ECC), 2011, pp. 4510-4515.

[2] Z. Qu, Cooperative Control of Dynamical Systems: Applications to Autonomous Vehicles. London: Springer, 2009.

[3] D. V. Dimarogonas and K. H. Johansson, "Further results on the stability of distance-based multi-robot formations," in American Control Conference, 2009, pp. 2972-2977.

[4] D. Sun, C. Wang, W. Shang, and G. Feng, "A synchronization approach to trajectory tracking of multiple mobile robots while maintaining time-varying formations,” IEEE Transactions on Robotics, vol. 25, no. 5, pp. 1074-1086, 2009.

[5] J. P. Desai, J. Ostrowski, and V. Kumar, "Controlling formations of multiple mobile robots," in IEEE International Conference on Robotics and Automation, vol. 4. IEEE, 1998, pp. 2864-2869.

[6] M. Cao, C. Yu, and B. Anderson, "Formation control using range-only measurements," Automatica, vol. 47, no. 4, pp. 776-781, 2011. 
[7] J. Cortes, S. Martinez, T. Karatas, and F. Bullo, "Coverage control for mobile sensing networks," IEEE Transactions on Robotics and Automation, vol. 20, no. 2, pp. 243-255, 2004.

[8] R. Olfati-Saber, "Flocking for multi-agent dynamic systems: Algorithms and theory," IEEE Transactions on Automatic Control, vol. 51, no. 3, pp. 401-420, 2006.

[9] H. G. Tanner, A. Jadbabaie, and G. J. Pappas, "Flocking in fixed and switching networks," Automatic Control, IEEE Transactions on, vol. 52, no. 5, pp. 863-868, 2007.

[10] R. Olfati-Saber and R. Murray, "Distributed cooperative control of multiple vehicle formations using structural potential functions," in IFAC World Congress, 2002, pp. 346-352.

[11] N. Moshtagh and A. Jadbabaie, "Distributed geodesic control laws for flocking of nonholonomic agents," IEEE Transactions on Automatic Control, vol. 52, no. 4, pp. 681-686, 2007.

[12] S. Adinandra, Hierarchical Coordination Control of Mobile Robots. Ph.D thesis, University of Eindhoven, The Netherlands, 2012.

[13] D. Kostic, S. Adinandra, J. Caarls, N. van de Wouw, and H. Nijmeijer, "Saturated control of time-varying formations and trajectory tracking for unicycle multi-agent systems," in IEEE Conference on Decision and Control, 2010, pp. 4054-4059.

[14] A. Sadowska, T. v. den Broek, H. Huijberts, N. van de Wouw, D. Kostić, and H. Nijmeijer, "A virtual structure approach to formation control of unicycle mobile robots using mutual coupling," International Journal of Control, vol. 84, no. 11, pp. 1886-1902, 2011.

[15] H. Taub and D. Schilling, Principles of Communication Systems. McGraw-Hill Higher Education, 1986.

[16] N. Sofra and K. Leung, "Estimation of link quality and residual time in vehicular ad hoc networks," in IEEE Wireless Communications and Networking Conference, 2008, pp. 2444-2449.

[17] O. Tekdas et al., "Robotic routers: Algorithms and implementation,” The International Journal of Robotics Research, vol. 29, no. 1, pp. 110-126, 2010.

[18] E. Frew, "Information-theoretic integration of sensing and communication for active robot networks," Mobile Networks and Applications, vol. 14, no. 3, pp. 267-280, 2009.

[19] C. Dixon and E. Frew, "Maintaining optimal communication chains in robotic sensor networks using mobility control," Mobile Networks and Applications, vol. 14, no. 3, pp. 281-291, 2009.

[20] F. Lucas and C. Guettier, "Automatic vehicle navigation with bandwidth constraints," in Military Communications Conference, Oct 31, 2010-Nov 3 2010, pp. 1423 -1429.

[21] H. Chen, P. Hovareshti, and J. Baras, "Opportunistic communications for networked controlled systems of autonomous vehicles," in Military Communications Conference, 2010, pp. 1430 -1435.

[22] J. Laneman, "Cooperative communications in mobile ad hoc networks," IEEE Signal Processing Magazine, vol. 23, no. 5, pp. 18-29, 2006.

[23] Z. Qu, J. Wang, C. Plaisted, and R. A. Hull, "Global-stabilizing near-optimal control design for nonholonomic chained systems," IEEE Transactions on Automatic Control, vol. 51, no. 9, pp. 1440-1456, 2006.

[24] H. Khalil, Nonlinear Systems. NJ: Prentice Hall, 2002.

[25] M. Krstic and H. Wang, "Stability of extremum seeking feedback for general nonlinear dynamic systems," Automatica, vol. 36, pp. 595-601, 2000. 